\title{
Adaptive Sharpening with Overshoot Control
}

\author{
Antonio Buemi, Gaetano Santoro, Arcangelo Bruna, and Mirko Guarnera
}

ST Microelectronics, AST Catania Lab, Imaging Group

Stradale Primosole, 50 - 95121 Catania, Italy

\{antonio.buemi, gaetano.santoro, arcangelo.bruna,

mirko.guarnera\} est.com

\begin{abstract}
This paper presents the Adaptive Sharpening with Overshoot Control (ASOC), an algorithm for digital image contrast enhancement. The ASOC exploits a properly defined band-pass filter in order to better discriminate the "uniform/not uniform" zones of the input (blurred) image. A more aggressive sharpening is then performed on the textured zones, whilst the homogeneous regions are preserved.
\end{abstract}

Keywords: sharpening, gradients, band pass filters, overshoot.

\section{Introduction}

The principal target of the sharpening in digital images [1] is to highlight fine details in an image or to enhance blurred images. Since not only edges or details but any discontinuity in the image could be enhanced by a trivial approach, one of the most important objectives in a sharpening algorithm design is providing sharpening without introducing noise amplification and the so-called ringing effects. Considering the typical horizontal profile of an edge (Fig.1), the target is making steeper such intensity profile. Several approaches have been presented in literature. The simplest algorithms (both conceptually and computationally) to the problem are the Unsharp Masking (UM) techniques [2],[3],[4]. Such methods are based on the idea of adding a high passed version $\mathrm{Z}$ of the original image $\mathrm{I}$ to the input image, yielding the enhanced image Y:

$$
Y(m, n)=I(m, n)+Z(m, n) \cdot \lambda
$$

where $\lambda$ is an "enhance factor", used in order to modulate the strength of the filter. Different methods of UM for image enhancement are compared in the same condition in [2]. The results of such analysis show that the majority of the UM algorithms are very sensitive to the enhancement factor $\lambda$ thus, for a good sharpening algorithm, this parameter must be adapted pixel by pixel with a recursive estimation, taking into account the statistics of neighboring pixel values. It increases the computational cost of the algorithm. An appropriate filter definition is a basic issue for the sharpening approach that uses sharpening masks in order to perform spatial filtering able to enhance the high contrast areas much more than the low contrast areas of the image. Using a filter properly defined, a good estimation of the local dynamics of the input 


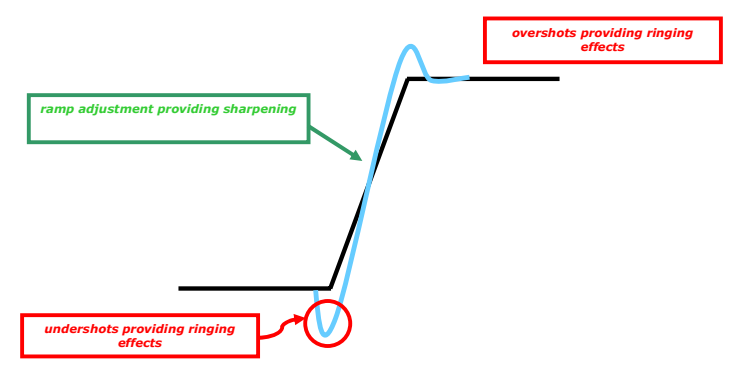

Fig. 1. Horizontal graylevel profile of an edge

image may be obtained performing an adaptive sharpening and avoiding noise amplification too. In [5], [6] are described several methods that exploit the Human Visual System properties and accurate filter definition in order to achieve contrast enhancement. An ad hoc sharpening filter definition method is described in [7].

The histogram equalization algorithms for contrast enhancement [1] represent a good tradeoff between simplicity and effectiveness. Global histogram equalization methods define a transform function depending on the whole image histogram information. Such transform allows stretching the contrast of high histogram region and compressing the contrast of the low histogram region. The simplicity of such procedure makes it appreciable for the efficiency of the algorithm, but limits the contrast improvement. The local histogram equalization approaches provide better results in terms of image quality because they allows to adapt the contrast strength to local brightness features of the input images, even if such improvement involves all pixels in the image and implies a consequent complexity grow. Nevertheless, the complexity could be reduced by splitting the histogram in partially overlapped subblocks and processing them independently [8]. Overlapping of such sub regions allow to avoid undesired blocking effects, too. Other approaches tried exploiting other image domains. A solution for sharpening compressed images in the Discrete Cosine Transform domain has been proposed in [9]. Such technique is applied to images compressed using the JPEG standard. The sharpening is achieved by suitably scaling each element of the encoding quantization table to enhance the high-frequencies of the image. The modified version of the encoding table is then transmitted in lieu of the original, thus the sharpening is performed without affecting compressibility. The wavelet domain is also suitable for sharpening algorithm development since allows local adaptation in smooth and non-smooth parts due to the theoretical link between wavelets and smoothness spaces, as discussed in [10]. Since conventional 2D wavelet transforms are separable, do they cannot represent non-separable structures of the image, e.g. directional curves, Nezhadarya et alii [11] proposed an innovative method to improve the sharpening of the input image using the so-called contourlet transform, whose directionality feature makes it suitable for representation of curves and edges in the image. Several solutions based on fuzzy logic have been also developed. In [11] a fuzzy bidirectional flow framework based on generalized fuzzy sets is proposed. A fuzzy operator is also defined in [13]. An adaptive fuzzy method for image contrast enhancement has been recently proposed in [14]. It is based on defining adaptively smooth and sharp parameters calculating the difference between the gray value of each pixel and the median of its neighborhood. The main goal of such approach is 
discerning among noisy, flat and high frequencies region of the image and joining noise reduction and contrast enhancement steps.

Traditional edge sharpening methods mainly increases the intensity differences across an edge, but the edge width remains unchanged, thus this kind of approaches produce only limited effects for wide and very blurred edges. An algorithm that performs the sharpening across the edge and also reduces the edge width is presented in [15]. The algorithm discussed in this paper is an efficient solution based on a $5 \times 5$ band-pass filter and designed to reduce the main drawbacks of most of the sharpening techniques: the noise amplification and the ringing effects. Since the contrast of edge in the image is usually achieved by raising the intensity of the brighter side of an edge and lowering the intensity of the darker side (Fig. 1), overshoots are introduced along both sides of the edges yielding the ringing effect. Solutions for this problem are also proposed in [16] and [17]. The algorithm ADSOC proposed in [17], in particular, is based on a $5 \times 5$ filter mask and it retrieves directions information to obtain a better sharpening along the edges. Our solution reduces the noise amplification thanks to a band-pass filter and using a simply noise control, whilst an over/undershoot control block has been developed in order to manage the ringing effect.

The rest of the document is organized as follows: the section 2 describes the ASOC; the section 3 presents an analysis of the algorithms performances in comparison with other techniques; the section 4 includes conclusions and final remarks.

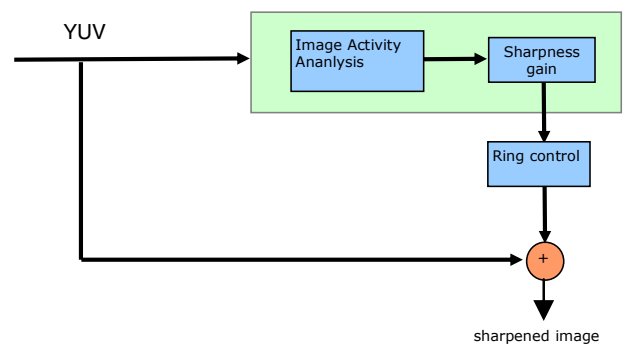

Fig. 2. Adaptive sharpening basic scheme

\section{Algorithm Description}

The Fig. 2 shows a simplified scheme of the Adaptive Sharpening with Overshoot Control (ASOC) algorithm. It consists on three basic steps:

- Image Activity Analysis: a band-pass filter is applied in order to discern the homogeneous and the textured regions of the image. A noise check is also enabled in order to avoid noise amplification.

- Sharpness Gain computation: the sharpness gain is the band-pass filter response that should be added to the input image in order to achieve sharpening.

- Ringing Control: an undershoot/overshoot control system is implemented in order to reduce unpleased ringing effects. 


\subsection{Image Activity Analysis}

This is the main step of the algorithm because it allows to find the zones of the image where the sharpening should be performed. Since the real borders and edges in images are not abrupt transitions but heavy gradients (due to optics and preprocessing on the camera), the ASOC uses Band Pass Filters. In fact this kind of filters is able to:

- $\quad$ highlight less the very high frequencies than the medium frequencies (border slopes);

- $\quad$ reduce the noise amplification;

- preserve gradients;

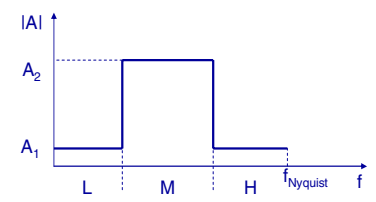

Fig. 3. Band Pass Filter frequency response

the noise amplification is reduced according to the following considerations. Let the image signal $\hat{s}(x, y)$ affected by a additive noise $\eta(x, y)$ be:

$$
\hat{s}(x, y)=s(x, y)+\eta(x, y)
$$

In the frequency domain it is:

$$
\hat{S}(u, v)=S(u, v)+N(u, v) .
$$

If we consider the signal as sum of band signal:

$$
S(u, v)=S_{L}+S_{M}+S_{H} \quad \text { and } \quad N(u, v)=N_{L}+N_{M}+N_{H} .
$$

Where $L, M$ and $H$ represent the Low, Medium and High Signal components.

Let now consider a pseudo-ideal HPF as shown in fig.3, with $\mathrm{A}_{2}>>\mathrm{A}_{1}$. If we apply the HPF to $\hat{s}(x, y)$, then:

$$
H P F \cdot \hat{S}=\left(S_{L}+N_{L}\right) A_{1}+\left(S_{M}+N_{M}\right) A_{2}+\left(S_{H}+N_{H}\right) A_{1} \cong\left(S_{M}+N_{M}\right) A_{2} .
$$

The Sharpening is normally:

$$
S_{\text {SHARP }}=\hat{S}+\alpha(H P F \cdot \hat{S}) \cong S+N+\alpha\left(S_{M}+N_{M}\right) A_{2} .
$$

In the Edge zones the $S_{M}$ signal has an amplitude greater than the $\mathrm{N}_{M}$ noise, then in edge zones:

$$
S_{\text {SHARP }} \cong S+N+\alpha A_{2} S_{M}
$$

In non edge zones the residual $A_{2} N_{M}$ can be minimized by using proper edge detection techniques. 
Fig. 4 shows the frequency response and the mask of the $5 \times 5$ band-pass filter used in the ASOC. Only the pixels of the images whose the filter response is greater than a given threshold $t h$ are processed, because they are assumed falling in textured regions of the image. This threshold resolve the problem of the above residual noise term. The main drawback of the thresholding is that some residual noise may remain, and thus highlighted. This noise is no more Gaussian, because it becomes "salt and pepper" and thus a median based analysis or morphological is required [18].

\begin{tabular}{|c|c|c|c|c|}
\hline eas? & Lasa & exise & Leava & -6004 \\
\hline tuxace & Daseo & Leset & ansos & - Anam \\
\hline Luse & atse & 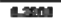 & D.sene & etiae \\
\hline-0.500 & onsury & LES & owerp & - the \\
\hline tang & Leasa & -611s & Lexag & $\tan \theta$ \\
\hline
\end{tabular}

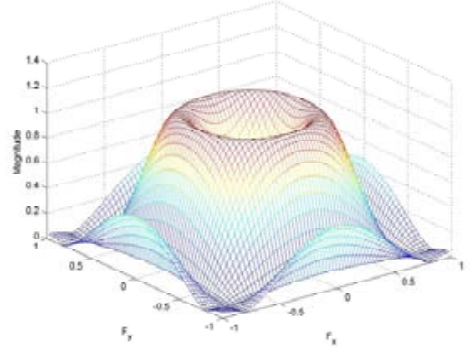

Fig. 4. The 5x5 band-pass filter used in the second step of the ASOC algorithm

We analyze the surrounding in order not to remove this "generated" noise but to identify it and do not highlight it through the HPF. For this reason we define a new noise level noise_th. For each pixel P under examination (Fig. 5), the minimum and the maximum of eight pixels of the $5 \times 5$ block are computed. If the condition:

$$
(\max -\min )<\text { noise_th } \quad \text { with } \operatorname{Max}=\max \{X 1, \ldots, X 8\}, \operatorname{Min}=\min \{X 1, \ldots, X 8\}
$$

is true, the luminance of the pixel P remains unchanged. Otherwise, the sharpening is performed.

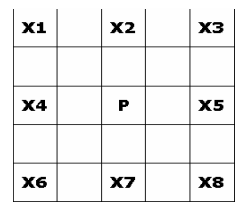

Fig. 5. $5 \times 5$ window used in the noise control block

When the noise control is activated, the number of isolated processed pixels decreases, whilst the quality of the final image increases. The improvement is much more visible in the enlarged detail showed in Fig. 6.

\subsection{Sharpness Gain Computation}

The ASOC mainly depends on two parameters:

Threshold: the region classification of the images depends on the chosen threshold, which affects the noise sensitivity of the algorithm according to a coring function. If 
the energy (that is the BFP applied to a pixel surrounding) is lower than this threshold, the pixel analyzed is left unsharpened.

The noise threshold can be a function of the estimated noise variance of the image [19]: Threshold $=f\left(\sigma_{\text {noise }}\right)$

Gain: sharpness boost. For each pixel $\mathrm{P}_{\text {in }}$, the sharpened value is given by:

$$
P_{\text {out }}=P_{\text {in }}+(\text { sharp } * \text { gain })
$$

Obviously the sharpness effect grows with the gain.

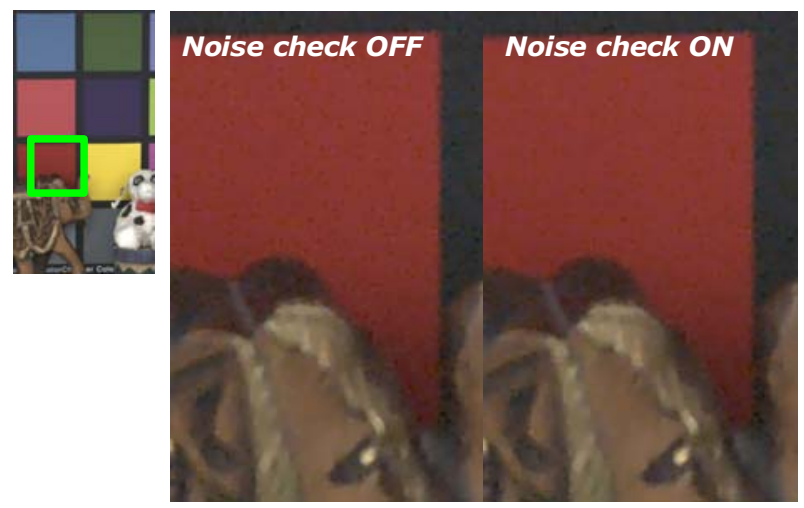

Fig. 6. Output of the ASOC with the noise check

\subsection{Ringing Control}

The under/overshoots control is performed exploiting the information concerning the local dynamic of the image which are used to reduce the sharpening side effects.

The value "sharp" to be added (for each pel) to the original luminance value $P_{i n}$ is computed as in the algorithm discussed in the previous section, that is the response of the band-pass filter computed by a mask of size $n \times n$.

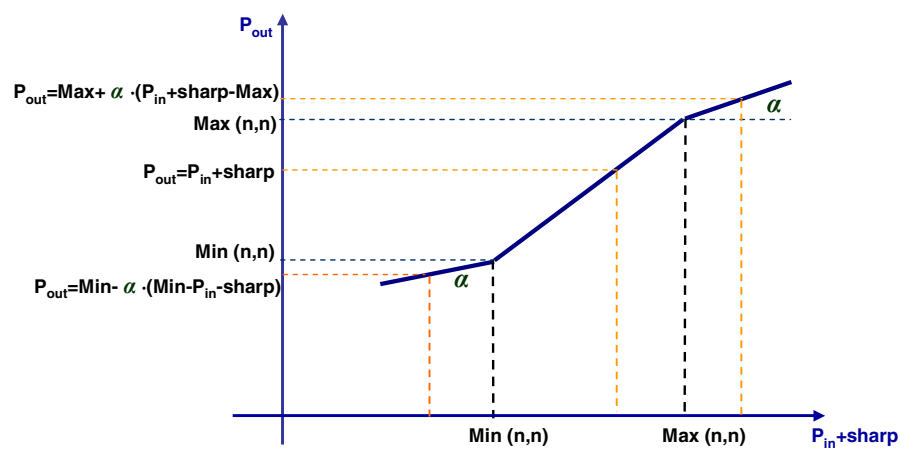

Fig. 7. Ringing effect control scheme 
If the sharpened value $P_{\text {out }}=P_{\text {in }}+$ sharp falls in the range $[$ Min,..., Max], with $\operatorname{Min}=\operatorname{minimum}(n, n)$ and $\operatorname{Max}=\operatorname{maximum}(n, n)$, it remains unchanged (Fig. 7). If $P_{\text {out }}$ is out of range, the overshot effect is reduced simply making smaller the gain between the sharpened value and the maximum (or minimum) of the block. The number of "control levels" $l$ could be fixed arbitrary. The "strength" of the overshot is the parameter that allows to vary the distance between the maximum/minimum value of the block and the sharpened value. The chart showed in Fig. 7 describes the ringing control geometry. Thus, the sharpened value Pout is given by:

$$
P_{\text {out }}=\left\{\begin{array}{clc}
\text { Max }+\alpha \cdot(\text { Pin }+ \text { sharp-Max }) & \text { if } & \text { Pin }+\operatorname{sharp~}>\operatorname{Max}(n, n) \\
\text { Pin }+ \text { sharp } & \text { if } & \text { Pin }+\operatorname{sharp~} \in[\operatorname{Min}(n, m), \operatorname{Max}(n, n)] \\
\text { Min- } \alpha(\text { Min-Pin-sharp }) & \text { if } & \operatorname{Pin}+\operatorname{sharp}<\operatorname{Min}(n, n)
\end{array}\right.
$$

where

$$
\alpha=\left\{\begin{array}{ccc}
0 & \text { if } & \text { strength }=0 \\
\frac{\text { strength }}{l} & \text { if } & \text { strength } \in[1, \ldots l]
\end{array}\right.
$$

Thus, if $\alpha=0$ then $P_{\text {out }}=$ Max and no overshot is created, whilst if is $\alpha=1$, the ringing control is disabled.

Observe that a slight ring makes the image more natural, whilst the absence of ringing may produce the unpleased effect of a flat image, thus in general is advised to enable a minimum overshoot.

\section{Experimental Results}

In order to evaluate the performances of the ASOC, the algorithm has been compared with several sharpening techniques. In particular, the following methods have been implemented and compared: AUM: Adaptive Unsharp Masking [3], RUM: Rational Unsharp Masking [4], ADSOC: Adaptive Sharpening with Overshoot Control [17].

The AUM is based on the idea of applying a different contrast level enhancement depending on the local dynamics of the images of interest. It employs a GaussNewton strategy to update the parameters needed to modify the contrast level and some Laplacian operators to estimate the local dynamics of the image. The RUM is a non-linear method that uses a rational control term to balance the enhancement intensity. It avoids noise amplifications using a rational control factor to measure the local activity level in the image in order to distinguish high, medium and low contrasted areas. The AUM algorithm yields a well-balanced sharpening effect at the expense of a high computational complexity, while the RUM overcomes this disadvantage, but it suffers from a minor flexibility. The ADSOC is based on a $5 \times 5$ High-pass filter and exploits directional information extracted by the response of a filters' bank in order to yield the sharpened image. It exploits some directional information in order to perform a stronger sharpening across the directions of the edges in the input image. In particular the response of a $5 \times 1$ band-pass filter banks 

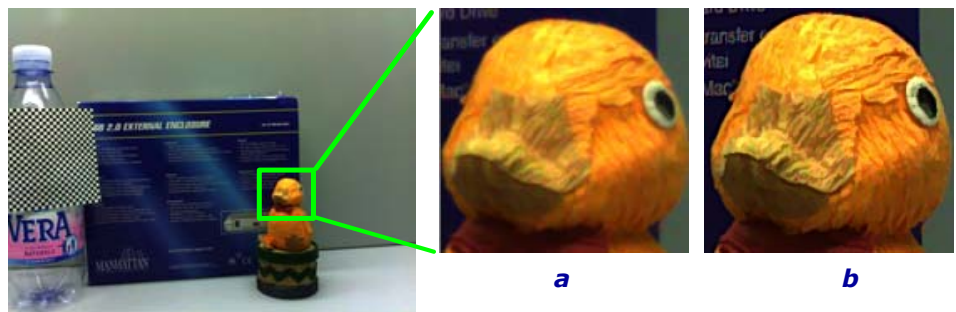

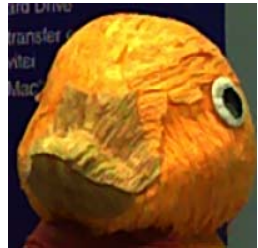

C

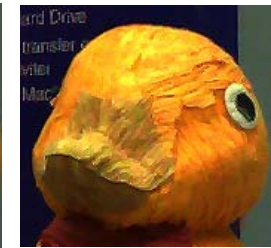

d

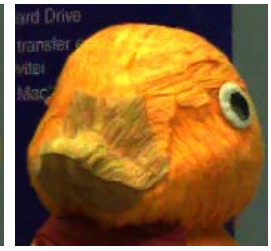

e

Fig. 8. Comparative tests (a) Input image. (b) Outputo of ADSOC. (c) Output of ASOC. (d) Output of the RUM. (e) Output of AUM.

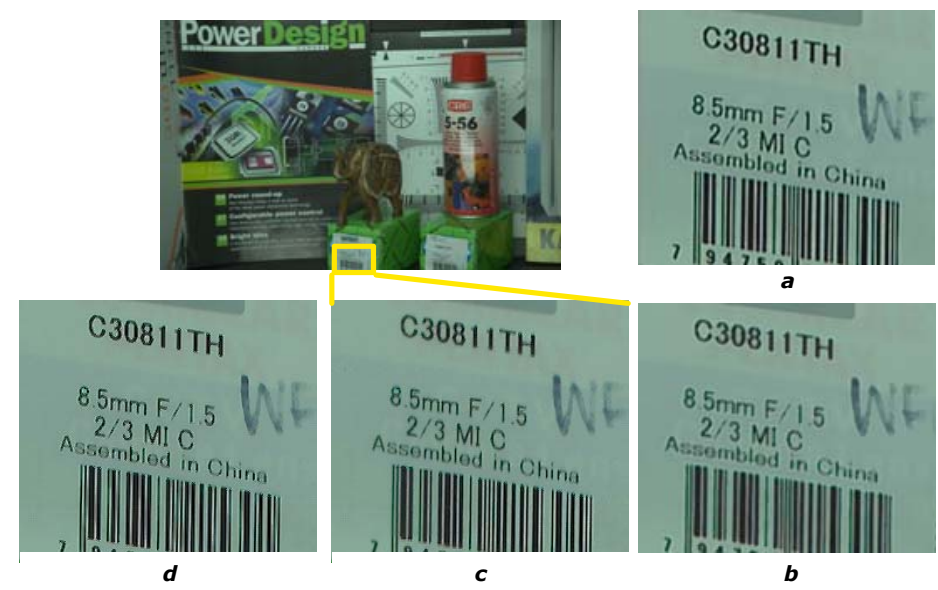

Fig. 9. (a) Image sharpened using ASOC. (b) Output of the ADSOC. (c) Output of the AUM. (d) Output obtained applying the RUM algorithm.

(see [17] for details) is used to compute the sharpness gain. If the pixel $p$ under examination yields at least a filter response $d_{i}$ greater than a fixed threshold $t h 2$, it corresponds to an edge region of the image, thus the intensity of $p$ is adjusted to achieve sharpening. The estimated gain to be added to $p$ is the response of the $5 \times 1$ filter corresponding to the direction orthogonal respect to $d_{i}$. The Fig. 8 shows comparative tests among the four algorithms described above. Note that both ADSOC and ASOC perform a strong sharpening, avoiding unpleasant ringing effects on the writing in the background. The RUM is not adaptive, so the contrast is enhanced indiscriminately in the whole image. The AUM algorithm allows to avoid ringing and artifacts, but the sharpening effect is reduced. Since the ringing effect is completely 
removed the image appears quite unnatural. Moreover the additional cost of the recursive process required by the AUM implies a large amount of additional resources, so it could be applied only in post-processing applications, whilst the other solutions are less expensive in terms of computational and memory cost.

The further example showed in Fig. 9 highlights the different performances of the ASOC and ADSOC in a critical case. The ADSOC introduces some artifact along the borders of the writing. Such undesired effect is produced by the directional tests that may give a different response for adjacent pixel, e.g. along a curve. A possible solution of this problem could be the use of high-pass filters in the directional test in order to obtain a more accurate response from the filters' bank. In the same example, the AUM provides a limited sharpening effect, the RUM introduces some unpleasant effect in the bottom of the picture.

\section{Conclusions}

An efficient sharpening algorithm has been presented. It is based on a band-pass filter able to discern uniform and detailed zones of the image in order to apply a balanced contrast enhancement. The proposed approach allows achieving sharpening with reduced noise amplification and ringing effect.

\section{References}

[1] Gonzales, R.C., Woods, R.E.: Digital Image Processing. 2, pp. 91-109/125-141. Prentice Hall, Englewood Cliffs (2002)

[2] Badamchizadeh, M.A., Aghagolzadeh, A.: Comparative Study Of Unsharp Masking Methods For Image Enhancement. In: Proceedings of the Third International Conference on Image and Graphics, ICIG 2004 (2004)

[3] Polesel, A., Ramponi, G., Mathews, V.J.: Image Enhancement via Adaptive Unsharp Masking. IEEE Trans. on Image Processing 9(3), 505-510 (2000)

[4] Ramponi, G., Polesel, A.: A Rational Unsharp Masking Technique. Journal of Electronic Imaging 7(2), 333-338 (1998)

[5] Baldrich, R., Vanrell, M., Benavente, R., Salvatela, A.: Color Image Enhancement Based On Perceptual Sharpening. In: Proceedings of International Conference on Image Processing (ICIP), September 14-17, vol. 3, pp. III-401-III-404 (2003)

[6] Marsi, S., Ramponi, G., Carrato, S.: Image Contrast Enhancement Using a Recursive Rational Filter. In: IEEE International Workshop on Imaging System and Techniques, Stresa, Italy (IST), May 14 (2004)

[7] Murphy, N.P.: Determining a Sharpening Filter for a Digital Image, European Patent, EP1411470A2, April 21 (2004)

[8] Kim, J.-Y., Kim, L.-S., Hwang, S.-H.: An Advanced Contrast Enhancement Using Partially Overlapped Sub-block Histogram Equalization. In: Proceedings of IEEE International symposium on Circuits and Systems (ISCAS 2000), May 28-31 (2000)

[9] Konstantinides, K., Bhaskaran, V., Beretta, G.: Image Sharpening in the JPEG Domain. IEEE Transactions On Image Processing 8(6) (June 1999)

[10] Berkner, K., Gormish, M.J., Schwartz, E.L., Bolielc, M.: A New Wavelet-Based Approach to Sharpening and Smoothing of Images in Besov Spaces with Applications to Deblurring, September 10-13, vol. 3, pp. 797-800 (2000) 
[11] Nezhadarya, E., Shamsollahi, M.B.: Image Contrast Enhancement by Contourlet Transform. In: 48th International Symposium ELMAR-2006, June 07-09 (2006)

[12] Fu, S.: Fuzzy Bidirectional Flow for Adaptive Image Sharpening. In: IEEE International Conference on Image Processing, ICIP 2005, September 11-14, vol. 1, pp. 917-920 (2005)

[13] Russo, F., Ramponi, G.: A Fuzzy Operator for the Enhancement of Blurred and Noisy Images. IEEE Transactions on Image Processing 4(8) (August 1995)

[14] Chen, D., Qian, Y.: An Improved Algorithm of Adaptive Fuzzy Image Enhancement. In: International Symposium on Computer Science and Computational Technology (2008)

[15] Chung, M.K., Kee, D., Leu, J.-G.: Edge sharpening through ramp width reduction. Image and Vision Computing 18(6), 501-514 (2000)

[16] Li, X.: Luminance Enhancement With Overshoot Reduction Control Based on Chrominance Information, US Patent 6453068, September 17 (2002)

[17] Buemi, A., Santoro, G., Bruna, A., Guarnera, M.: Adaptive Directional Sharpening with Overshoot Control. Electronic Imaging, January 27-31 (2008)

[18] Wang, Z., Zhang, D.: Progressive switching median filter for the removal of impulse noise from highly corrupted images. Circuits and Systems II: IEEE Trans on Analog and Digital Signal Processing 46(1), 78-80 (1999)

[19] Bruna, A., Bosco, A., Smith, S., Tomaselli, V.: Fast noise level estimation using a convergent multiframe approach. In: IEEE International conference on image processing (ICIP 2006), October 8-11 (2006) 